An average reduction of $50.5 \%$ was observed in the affected area, a reduction of $24.1 \%$ in the baseline pain score, using a Visual Analogue Scale (VAS), and a reduction of $12 \%$ in the peak pain score.

Functional capacity had limited improvement.

The most common side effects were application site reactions including intense burning, pain, swelling and erythema. Blood pressure alterations were not noticed.

Conclusions Taking into account the 'IMMPACT' recommendations [2], the observed reduction in the VAS score was lower than $30 \%$ both in peak and baseline score. This did not translate into a clinically significant improvement.

Given the size of the study sample, the conclusions although interesting, must be confirmed with additional data.

In times of severe budget restraints, heath care providers must take into account both the benefits that new treatments bring to patients and the limited resources available in public services.

No conflict of interest.

\section{CPC-055 EVALUATION OF TREATMENT COMPLIANCE IN MULTIPLE SCLEROSIS PATIENTS AND ITS IMPACT ON THE CLINICAL STABILISATION OF THE DISEASE}

doi:10.1136/ejhpharm-2013-000276.512

Al Rodrigues, AF Fernandes, R Marques. Centro Hospitalar Leiria-Pombal E.P.E., Pharmacy Department, Leiria, Portugal

Background Multiple sclerosis is a degenerative disease in which compliance with therapeutic regimens is extremely important in the clinical stabilisation of disease.

Purpose To evaluate the compliance of patients with treatment and the impact it has on the clinical stabilisation of the disease.

Materials and Methods Data were collected using a patient survey and consulting the hospital computer system. Statistical analysis was done with SPSS. The following data were collected: number of patients, average age, number of outbreaks and management failures in the last six months, reason for failure and flaws in the administration of medicines reported in the survey.

Results The sample (97 patients, mean age $41.73 \pm 9.37$ years old) was not only representative of the total MS patients followed in Centro Hospitalar Leiria-Pombal (CHLP) but also of the epidemiological data on the disease.

Over the past six months, $18.6 \%$ of patients had at least one outbreak. Regarding administration failures, $24.7 \%$ of patients admitted to failing to administer their medicines at least once, $45.8 \%$ of these failed more than three times.

The main reason for failing to administer the treatment was patient oversight and that represented $48 \%$ of total failures.

The largest number of administration failures was observed in patients treated with Interferon B $1 \mathrm{~b} 8 \mathrm{MIU}$ (66.7\%).

$86.6 \%$ of patients had administered their medicine correctly.

In this group of patients, there is no statistically meaningful correlation between the failures of management and inventory, with the number of outbreaks that occurred.

Conclusions Generally, multiple sclerosis patients followed in CHLP are a group with a great commitment to following their treatment.

There was a significant percentage of people who failed to administer all doses correctly, although there is no statistically meaningful correlation with the number of outbreaks that occurred, and there was a significant incidence of outbreaks in the last 6 months, suggesting that the disease has a multifactorial nature.

As I see it, the pharmaceutical staff plays an essential role in promoting compliance, which is crucial for stabilising the patients' clinical condition.

No conflict of interest.

\section{CPC-056 EVOLUTION IN DRUG RELATED PROBLEMS IDENTIFIED IN PHARMACIST NOTES AT THE EMERGENCY DEPARTMENT, HILLER $^{-}$D HOSPITAL DENMARK}

doi:10.1136/ejhpharm-2013-000276.513

'DV Tomsen, 'MB Jensen, 'LG Gammelgaard, '2 Christrup. 'Region Hovedstadens Apotek, Apoteksenhed Nord, Hilleroed, Denmark; 2University of Copenhagen, Pharmacology, Copenhagen, Denmark

Background 'Pharmacists in the Emergency Department' is a twoyear implementation project carried out in collaboration between the pharmacy of Capital Region and the Emergency Department (ED) at Hillerød hospital. The task of the pharmacist is to draw up a current and valid medicines history and to make a medicines review before the physician sees the patient at the ED. During the first year of the project the interventions developed gradually while the professional skills and clinical experience of the pharmacists built up.

Purpose To describe the evolution of the interventions recommended when Drug Related Problems (DRPs) are identified, as described in the pharmacist's notes.

Materials and Methods 5 samples of pharmacist's notes were recorded. The samples represent the interventions made in the 2 first weeks of each quarter of 2011 and the first quarter of 2012. This showed the development in interventions made by pharmacists. The interventions were coded based on 8 categories of DRP introduced by Hepler and Strand. In total 383 pharmacist's notes were analysed.

Results In all 549 DRPs were identified. $70-80 \%$ of the pharmacist's notes contained one or more DRP. On average 1.4 DRPs were identified per note. During the first 15 months of the project the DRPs recorded evolved as follows: The number of comments tended to increase in the categories "inappropriate choice of drug", "overdose", "adverse drug events" and "medicine without reasonable indication". The number of comments identified in the category "interactions" decreased. The categories "untreated indication", "subtherapeutic dosing" and "inappropriate use by the patient" were stable throughout the study period.

Conclusions When introducing a new pharmaceutical service one must expect a gradual evolution of the interventions as the pharmacist gradually develops hands-on-competencies and clinical experience on the particular ward. After 12 months, the findings in the pharmacist notes were stable. This must be taken into account when introducing new pharmaceutical services in the clinic.

No conflict of interest.

\section{CPC-057 EVOLUTION OF CLINICAL TRIAL PRESCRIBING INCIDENTS}

doi:10.1136/ejhpharm-2013-000276.514

'I Larrodé, 'A Idoipe, ${ }^{2}$ S Bernabé, 10 Pascual, 'M Uriarte, '1 Real. 'Servet, Pharmacy, Zaragoza, Spain; 'Instituto Aragones Ciencias Salud, Pharmacy, Zaragoza, Spain

Background It is essential to record incidents in clinical trials (CT) to monitor them appropriately. It is a basic tool to analyse and detect problems.

Purpose To analyse the development in prescription incidents recorded from 2009 to 2011, to identify and resolve quality problems, with the aim of establishing corrective actions to reduce CT problems in a process of continual improvement.

Materials and Methods The most frequent incidents were found in the prescription phase. Data were recorded using the following items: date, person reporting, CT identification, department, professional involved, description of the problem and corrective measures. The evolution of incidents was analysed by chi square.

Results 186 events were recorded in a total of 331 CTs. The most frequent events occurred mainly in the prescription phase $(49.0 \%)$ 